\title{
BACKGROUND IN A TEST OF DETECTING “COOPERATIVE” PARAPOSITRONIUM ANNIHILATION BY THE 32-CRYSTAL SPECTROMETER ARGUS*
}

\author{
Nevenka M. Antovići ${ }^{* *}$, Sergey K. Andrukhovich², Alexandr V. Berestov ${ }^{2}$ \\ ${ }^{1}$ University of Montenegro, Faculty of Natural Sciences and Mathematics, Podgorica, Montenegro \\ ${ }^{2}$ National Academy of Sciences of Belarus, Institute of Physics, Minsk, Belarus
}

\begin{abstract}
Parapositronium - a singlet positronium ground state, has the total angular momentum of electron and positron forming the atom $J_{s}=o$, magnetic quantum moment $m=o$, and its annihilation spectrum is dominantly created by the $511 \mathrm{keV}$ discrete photons. A process of cooperative emission of annihilation photons (cooperative annihilation) by a system of positronium atoms, i.e., annihilation superradiance, had been considered by other researchers, and the theory of annihilation superradiance in a system of parapositronium atoms for two-photon annihilation was constructed (two interacting parapositronium atoms; emission of the $1022 \mathrm{keV}$ photons flying apart at an angle of $180^{\circ}$ ). The 32-crystal spectrometer ARGUS, with 16 detector pairs at an angle of $180^{\circ}$ capable of registering double gamma coincidences, with lead collimators ( $80 \mathrm{~mm}$ in diameter) mounted on each detector - was used to test the phenomenon. Parapositronium annihilation spectra were acquired using ${ }^{22} \mathrm{Na}\left(\mathrm{A}=4 \cdot 1 \mathrm{O}^{5} \mathrm{~Bq}\right)$ as a positron source, and $\mathrm{SiO}_{2}$ (as „positronium forming“ medium; probability: 32 \%), as well as Al (as „positronium not forming" target) used as a blank - for estimation of the background events. In the case when after emission of two starting positrons from ${ }^{22} \mathrm{Na}$ (i.e., the $1275 \mathrm{keV}$ nuclear photons) coincident registration (respecting the spectrometer time resolution) of four annihilation photons should be considered as a six-fold coincidence event, experimentally obtained counting rates were $-0.25 \mathrm{~s}^{-1}\left(\mathrm{SiO}_{2}\right)$ and $0.23 \mathrm{~s}^{-1}(\mathrm{Al})$, while theoretically predicted $-0.34 \mathrm{~s}^{-1}$. The main background process competitive to a registration of the parapositronium cooperative annihilation is four-fold coincidence event (the two $180^{\circ}$ detectors register $1275 \mathrm{keV}$ photons, and two - summing of the $511 \mathrm{keV}$ annihilation photons), with experimental counting rates $-0.026 \mathrm{~s}^{-1}\left(\mathrm{SiO}_{2}\right)$ and $0.023 \mathrm{~s}^{-1}(\mathrm{Al})$, as theoretically predicted $-0.023 \mathrm{~s}^{-1}$. On the other hand, cooperative annihilation should be a four-fold coincidence event (the two $180^{\circ}$ detectors register $1275 \mathrm{keV}$ photons, and two - $1022 \mathrm{keV}$ photons), which has not been registered by the ARGUS spectrometer (theoretically predicted counting rate - 1.7.1 $\mathrm{O}^{-5} \mathrm{~s}^{-1}$ ). The analyses showed that probability of detecting the parapositronium cooperative annihilation will increase significantly with increasing positron source activity, but also with decreasing diameter of lead collimators.
\end{abstract}

Key words: spectrometer ARGUS, parapositronium, cooperative annihilation, background

DOI: $10.21175 / \operatorname{RadJ} .2016 .01 .06$

\section{INTRODUCTION}

Positronium (Ps) - an exotic atom consisting of electron and positron, has two ground states: a singlet or para-Ps $\left[{ }^{\mathrm{SPs}}\left({ }^{1} \mathrm{~S}_{0}\right)\right.$ or $p$-Ps], and a triplet or ortho-Ps $\left[{ }^{t} \mathrm{Ps}\left({ }^{3} \mathrm{~S}_{1}\right)\right.$ or $o$-Ps]; with lifetime in vacuum $-1.25 \cdot 10^{-10} \mathrm{~S}$ and $1.4 \cdot 10^{-7} \mathrm{~s}$, respectively [1]. These states are different in regard to the total angular momentum of electron and positron forming the atom $-\mathrm{J}_{\mathrm{s}}=\mathrm{O}$ in the case of $p$ Ps (electron and positron spins are antiparallel, and magnetic quantum moment: $\mathrm{m}=0$ ), and $\mathrm{J}_{\mathrm{t}}=1$ in the case of $o$-Ps (electron and positron spins are parallel, and three sub-states different in magnetic quantum moment: $\mathrm{m}=+1,0,-1$, are possible). The $p$-Ps experiences $2 \gamma$-annihilation (the $511 \mathrm{keV}$ discrete photons flying apart at an angle of $180^{\circ}$ ), while $o$-Ps $3 \gamma$-annihilation (continuous energy spectrum in the range from o to $511 \mathrm{keV}$ ) [1].

Experimental research on Ps atom was basically related to the energy spectrum of annihilation photons, coincidences of $3 \gamma$-decay photons, coincidence rates of
$2 \gamma$-decay photons as a function of angles between two detectors, delayed coincidences - positron emission and annihilation radiation [1], rare Ps decays - on $4 \gamma$ $(p$-Ps) and $5 \gamma$-rays (o-Ps) [2], asymmetric $3 \gamma$-decay [3], etc. This atom (i.e., its decay) has been also used to test, for example, CPT-invariance [e.g., 4, 5, 6].

Despite the fact that annihilation of Ps atoms (in free space, but also in various media) has been studied in considerable details, there are theoretically predicted phenomena related to Ps-annihilation which have not been experimentally confirmed (detected) so far.

This study considers possibility to detect annihilation photons from "cooperative" annihilation of $p$-Ps, using the 32-detector coincidence spectrometer ARGUS (Institute of Physics, National Academy of Sciences of Belarus, Minsk).

\footnotetext{
${ }^{*}$ The paper was presented at the Third International Conference on Radiation and Applications in Various Fields of Research (RAD 2015), Budva, Montenegro, 2015.

Contact: antovicn@yahoo.com
} 


\section{BASICS OF THE P-PS COOPERATIVE ANNIHILATION THEORY}

The process of cooperative emission of annihilation photons (cooperative annihilation) by a system of Ps atoms, i.e., annihilation superradiance, had been considered in a few works [e.g., 7, 8].

Using the concepts of Dicke's theory of optical supperradiance in a system of two-level atoms, as well as in a system of excitations, the authors of ref. [7] (emphasizing important differences between optical and annihilation superradiance) constructed a theory of annihilation superradiance in a system of $p$-Ps atoms - for $2 \gamma$-annihilation. Based on interaction of the Ps atoms through the field of virtual photons (quantum electrodynamic effect of the fourth order), an equation of the annihilation radiation propagation in a Ps-medium was developed [7].

Considering electron-positron interaction as nonradiative in nature, and studying the radiative interactions of a Ps atom with the field of its own and external photons, it was concluded that a field changes significantly not only the energies, but also lifetime of the Ps atom states [8]. That is then used for the study of the annihilation decay kinetics of a $p$-Ps atom from two states participating in stimulated optical transitions excited by a laser [9] (while two-, three-, and six-photon cross sections and ionization rates for Ps at laser frequencies of experimental interest were also calculated [10]; and Ps in a laser field was considered extensively [e.g., 11]).

So, cooperative annihilation implies a bound state of two interacting $p$-Ps atoms, which decays through emission of two $1022 \mathrm{keV}$ photons flying apart at an angle of $180^{\circ}$

$\mathrm{sPs}+\mathrm{sPs} \rightarrow \mathbf{2 \gamma}$.

If a volume (of gas, for example) under investigation contains $\mathrm{N}$ positrons, it can be taken that $\mathrm{N}_{\mathrm{p}}$ of them annihilate without Ps forming (a free annihilation into two $511 \mathrm{keV}$ photons), and that $\mathrm{N}_{p-\mathrm{Ps}}$ and $\mathrm{N}_{o-\mathrm{Ps}}$ are numbers of $p$ - and $o$-Ps atoms, respectively.

According to the prediction (O. N. Gadomskii, as discussed in [12]), probability ratio: cooperative versus free annihilation of $p$-Ps is

$$
\frac{\mathrm{P}_{\mathrm{Coop}}}{\mathrm{P}\left({ }^{\mathrm{s}} \mathrm{Ps}\right)} \approx 5 \cdot 10^{-5}
$$

If a sample is placed between two detectors (at an angle of $180^{\circ}$ ) registering double coincidences of the $1022 \mathrm{keV}$ photons, counting rate of the events corresponding to the $p$-Ps cooperative annihilation can be

$$
\mathrm{N}_{\text {effect }} \sim \mathrm{N}_{p-\mathrm{Ps}} \varepsilon_{1022}^{2} \Omega \mathrm{P}_{\text {Coop }}
$$

where $\varepsilon_{1022}$ represents one-detector photoefficiency for the $1022 \mathrm{keV}$ photon (assuming that detectors have the same registration efficiency); $\Omega$ is a solid angle from the source/target to the detector (there is a strong angular correlation between the decay's photons: $180^{\circ}$ ).

Taking that $\left(\mathrm{N}_{\mathrm{p}}+\mathrm{N}_{p \text {-Ps }}\right)$ positrons give the decay into two $511 \mathrm{keV}$ photons, it is possible to estimate the main background process. This process presents a registration (by two-detector system) of four $511 \mathrm{keV}$ photons, which are products of two independent decays of $p$-Ps atoms (or free positron annihilation; or a combination of these processes) and due to the summing effect competitive to the photons from cooperative annihilation, i.e.,

$\mathrm{N}_{\text {background } \sim}\left(\mathrm{N}_{\mathrm{p}}+\mathrm{N}_{p-\mathrm{Ps}}\right) \varepsilon_{511}^{4} \Omega^{2}$,

where $\varepsilon_{511}$ is one-detector photoefficiency for the 511 keV photon.

From Eqs. (3) and (4), the effect/background ratio would be

$$
\frac{\mathrm{N}_{\text {effect }}}{\mathrm{N}_{\text {background }}} \approx \frac{\mathrm{N}_{p-\mathrm{Ps}} \mathrm{P}\left({ }^{\mathrm{s}} \mathrm{Ps}\right) \cdot 5 \cdot 10^{-5} \varepsilon_{1022}^{2}}{\left(\mathrm{~N}_{\mathrm{p}}+\mathrm{N}_{p-\mathrm{Ps}}\right) \varepsilon_{511}^{4} \Omega}
$$

\section{SPECTROMETER ARGUS}

The spectrometer of $\gamma$-coincidences ARGUS consists of 32 sections (12 penta- and 20 hexahedral pyramids), i.e., $32 \mathrm{NaI}(\mathrm{Tl})$ detectors (D) of (15 x 10) $\mathrm{cm}^{2}$ [13]. Its total solid angle is $\sim 0.9 \cdot 4 \pi \mathrm{sr}$, while the time resolution $(\tau)$ for two-fold coincidences $(\mathrm{k}=2)$ is $(45 \pm 5) \mathrm{ns}$ at $100 \%$ efficiency of registration [12]. There are 496 detector-duplet combinations capable of registering double gamma coincidences (among them, 16 are at an angle of $179.99^{\circ}$, i.e., $\sim 180^{\circ}-$ see, for example [14]).

It is maintained in a low background room and shielded by concrete and lead. A special container consisting of a lead pyramid with walls up to $6 \mathrm{~cm}$ thick, as well as a $10 \mathrm{~cm}$-thick disc, shields each of the detectors. Additionally, to reduce the probability of detecting Compton-scattered photons, a disc-shaped lead collimator (thickness $30 \mathrm{~mm}$, and orifice diameters $80 \mathrm{~mm}$ - side facing the detector surface, and $70 \mathrm{~mm}$ - opposite site) was mounted on each detector (see, for example [14]).

An outside overview of the ARGUS mechanical construction is illustrated in Fig. 1a, while a detector assembly - in Fig. 1b. The detectors are denoted from 1 to 32 such that the number for one detector and the number for the detector on the opposite side differ by 16, simplifying the spectrometric data analyses. A counting mode can be integral $(\mathrm{k} \geq 1)$ or coincidence (with multiplicities from 1 to 5 ).

The setup has modular pulse-processing electronics made in the CAMAC standard, and is operated online with a personal computer (with several program packages available - for processing various spectrometric datasets $[12,13])$. 


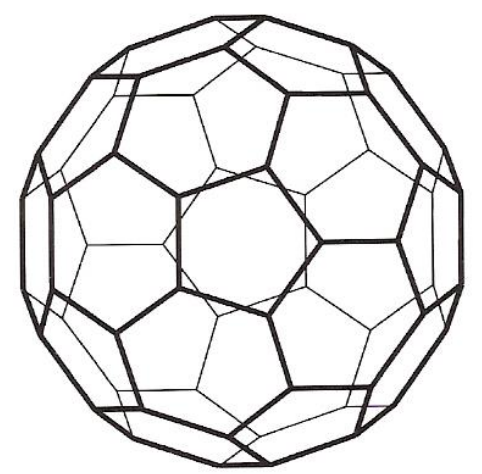

a)

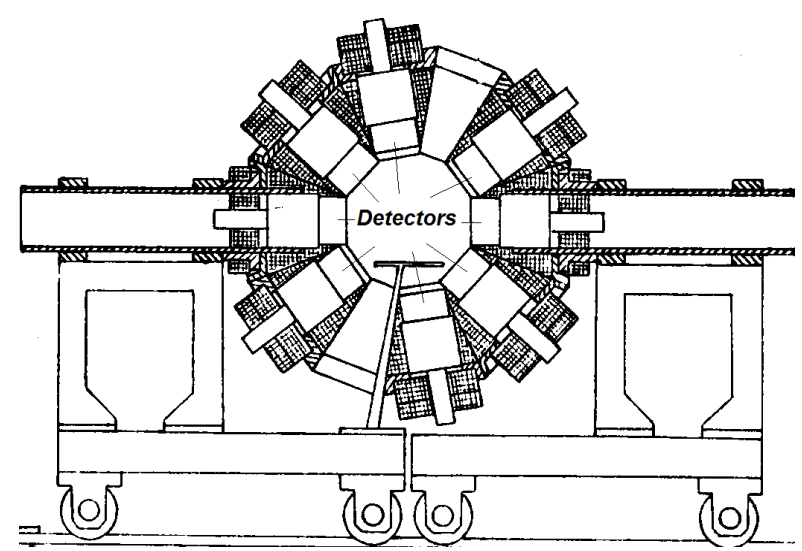

b)

Figure 1. ARGUS schematic: outside overview of the mechanical construction (a), detector assembly (b)

A schematic of the ARGUS system has been reported before (see, for example [14]), showing $\mathrm{NaI}(\mathrm{Tl})$ detectors, time discriminators by the leading edge, delay univibrators, majority coincidence circuit operating by pulse overlapping and permitting the selection of a coincidence fold between 1 and 5 , analogto-digital converters, hodoscope, splitter-univibrator, counter of events, clock-pulse generator, live-time counter, CAMAC crate controller, strobe pulses for the $\mathrm{ADC}$, strobe pulses for the hodoscope, signal following the input strobe pulse from the majority coincidence circuit, dead-time signal, clock pulses. Basically, for each detected coincidence, the numbers of detectors registering the event are recorded, as well as the photon energies.

This spectrometer was used to test the $p$-Ps cooperative annihilation, i.e., to test possibility for detecting abovementioned photons (two, flying apart at an angle of $180^{\circ}$, both with an energy of $1022 \mathrm{keV}$ ) if ${ }^{22} \mathrm{Na}$ is a positron source. During its decay, emission of a positron is accompanied by the emission of a nuclear photon with an energy of $1275 \mathrm{keV}$.

Namely, research on Ps-annihilation was often performed by means of ${ }^{22} \mathrm{Na}$ and "Ps-forming" (such as $\mathrm{SiO}_{2}$ ) and "Ps-not forming" (such as Al) substances [e.g., 12, 15], and they were also used in the experiment presented here.

\section{THE EFFECT/BACKGROUND RATIO}

It is important to note that the $511 \mathrm{keV}$ photon probability (i.e., probability of its photopeak registration) for the ARGUS spectrometer was [12]

$\mathrm{P}_{511}=32 \varepsilon_{511} \Omega=0.13$,

where $\varepsilon_{511}=0.63$ is one-detector photoefficiency for the photon with energy of $511 \mathrm{keV}$, and $\Omega=0.0064$ - solid angle from the system's geometric center to individual detectors.

Analogously,

$\mathrm{P}_{1022}=0.107$,

in the view of $\varepsilon_{1022}=0.52$.

On the other hand, if ${ }^{22} \mathrm{Na}$ is used as a positron source the $1275 \mathrm{keV}$ photon probability is also important, and in the case of the ARGUS spectrometer it was

$\mathrm{P}_{1275}=0.1$,

since $\varepsilon_{1275}=0.49$.

Thus, by using ${ }^{22} \mathrm{Na}$ and assuming that the 1275 $\mathrm{keV}$ photon should be registered in a photopeak (in order to eliminate effects of scattered radiation), making events selection by the energy criteria (software ARGUS [12]) and taking into account the detectors energy resolution, processes that could obstruct a detection of cooperative annihilation have been considered. The source activity $\mathrm{A}=4 \cdot 10^{5} \mathrm{~Bq}$, time resolution $\tau=45 \mathrm{~ns}$, and the Ps formation probability $\mathrm{P}$ $\approx 40 \%$, were taken into account. The statistical weight of the triplet state is three times as great as that of the singlet one, i.e., the para-state is formed in $25 \%$ and the triplet-state in $75 \%$ of the cases involving the Ps formation [1], then $\mathrm{P}(\mathrm{sPs}) \approx 10 \%$.

Firstly, after emission of two starting positrons (i.e., the $1275 \mathrm{keV}$ nuclear photons) coincident registration of four annihilation photons can be considered as a sixfold coincidence event $[(1275) \times(1275) \times(511 \uparrow) \times(511 \downarrow) \times(511 \rightarrow) \times(511 \leftarrow)] \quad$ shown in Fig. 2.

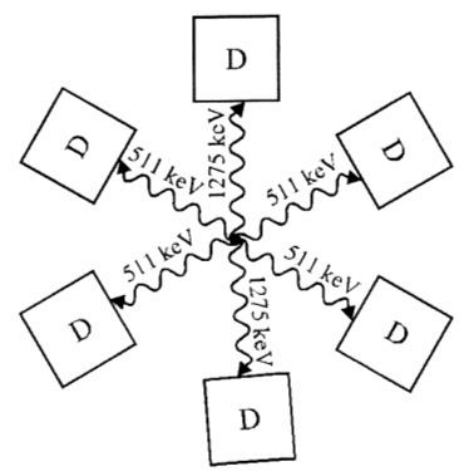

Figure 2. A six-fold coincidence event

The spectrometer ARGUS could register these events with the counting rate 


$$
\begin{aligned}
& \mathrm{N}_{6}=\mathrm{P}_{511} \frac{\mathrm{P}_{511}}{32 \Omega} 30 \frac{\mathrm{P}_{511}}{32} \frac{\mathrm{P}_{511}}{32 \Omega} 28 \frac{\mathrm{P}_{1275}}{32} 27 \frac{\mathrm{P}_{1275}}{32} \mathrm{~A}^{2} \tau= \\
& =\frac{30 \cdot 28 \cdot 27 \cdot \mathrm{P}_{511}^{4} \mathrm{P}_{1275}^{2}}{32^{5} \Omega^{2}} \mathrm{~A}^{2} \tau \approx 0.34 \mathrm{~s}^{-1} .
\end{aligned}
$$

At the same time, the main background process competitive to a registration of the $p$-Ps cooperative annihilation is a four-fold coincidence event $[(1275) \times(1275) \times(511 \uparrow+511 \rightarrow) \times(511 \downarrow+511 \leftarrow)]$. It means that detectors in a pair (at an angle of $180^{\circ}$ ) register random coincidences of the $511 \mathrm{keV}$ photons summing (Fig. 3).

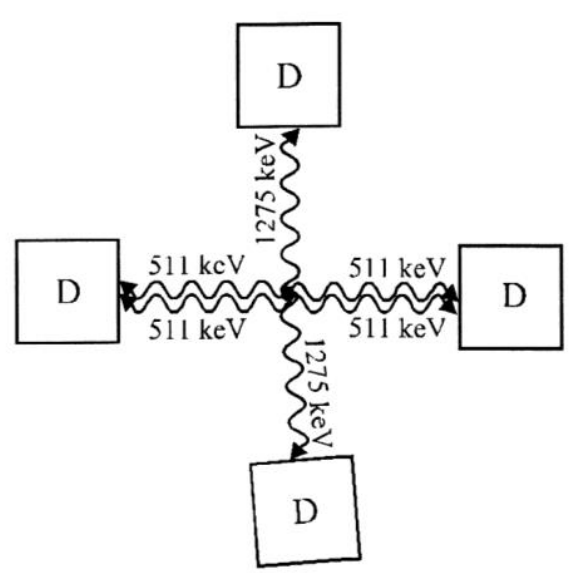

Figure 3. The main background process: four-fold coincidence event

The ARGUS spectrometer's counting rate of these background events would be

$$
\begin{aligned}
& \mathrm{N}_{\text {background }}=2\left(\mathrm{P}_{511} \frac{\mathrm{P}_{511}}{32 \Omega} \frac{\mathrm{P}_{511}}{32} \frac{\mathrm{P}_{511}}{32 \Omega}\right) 28 \frac{\mathrm{P}_{1275}}{32} 27 \frac{\mathrm{P}_{1275}}{32} \mathrm{~A}^{2} \tau=\text { (10) } \\
& =\frac{2 \cdot 28 \cdot 27 \cdot \mathrm{P}_{511}^{4} \mathrm{P}_{1275}^{2}}{32^{5} \Omega^{2}} \mathrm{~A}^{2} \tau \approx 0.023 \mathrm{~s}^{-1} .
\end{aligned}
$$

On the other hand, the $p$-Ps cooperative annihilation should be registered as a four-fold coincidence event [(1275)x(1275)x(1022 $\uparrow) \times(1022 \downarrow)]$ illustrated in Fig. 4.

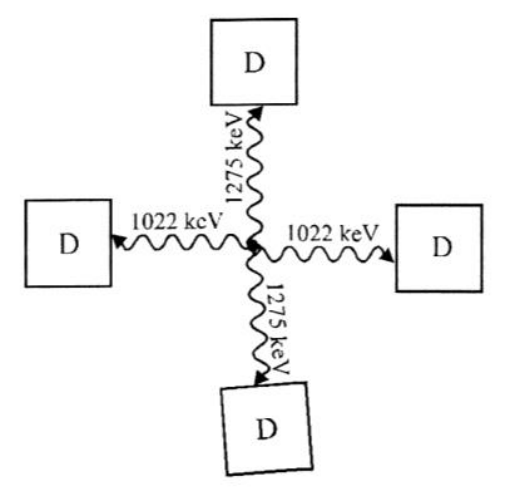

Figure 4. A cooperative annihilation of $p$-Ps

The effect counting rate is

$$
\begin{aligned}
& \mathrm{N}_{\text {effect }}=\mathrm{P}_{1022} \frac{\mathrm{P}_{1022}}{32 \Omega} 30 \frac{\mathrm{P}_{1275}}{32} 29 \frac{\mathrm{P}_{1275}}{32} \mathrm{~A}^{2} \tau \mathrm{P}\left({ }^{\mathrm{s}} \mathrm{Ps}\right) \cdot 5 \cdot 10^{-5}= \\
& =\frac{\mathrm{P}_{1022}^{2} \mathrm{P}_{1275}^{2} 30 \cdot 29}{32^{3} \Omega} 0.1 \cdot 5 \cdot 10^{-5} \mathrm{~A}^{2} \tau \approx 1.7 \cdot 10^{-5} \mathrm{~s}^{-1} .
\end{aligned}
$$

Therefore, under given conditions the ratio effect/background is around $0.07 \%$.

In order to test these data, Ps-annihilation spectra were acquired by the ARGUS spectrometer using ${ }^{22} \mathrm{Na}$ with an activity of $4 \cdot 10^{5} \mathrm{~Bq}$. This source was put inside $\mathrm{SiO}_{2}$ (as „Ps-forming“ medium, with $P \approx 32 \%$ ), as well as $\mathrm{Al}$ (as „Ps-not forming“ target) which is used as a blank - for investigation of the background events. The samples were positioned in the spectrometer's center, and then measured over 7500 s. Experimental counting rates $N$ (in $\mathrm{SiO}_{2}$ and $\mathrm{Al}$ ), as well as their theoretical values obtained using Eqs. (9) - (11), are given in Table 1.

Table 1 Counting rate of the coincident events spectrometer ARGUS

\begin{tabular}{|c|c|c|c|}
\hline $\begin{array}{c}\text { Coincident } \\
\text { event }\end{array}$ & $\begin{array}{c}N_{\mathrm{SiO} 2} \\
{\left[\mathrm{~s}^{-1}\right]}\end{array}$ & $\begin{array}{c}N_{A l} \\
{\left[\mathrm{~s}^{-1}\right]}\end{array}$ & $\begin{array}{c}N_{\text {theor }} \\
{\left[\mathrm{s}^{-1}\right]}\end{array}$ \\
\hline $\mathrm{k}=6$ & $0.25 \pm 0.02$ & $0.23 \pm 0.02$ & 0.34 \\
\hline $\begin{array}{c}\text { Background } \\
(\mathrm{k}=4)\end{array}$ & $0.026 \pm 0.006$ & $0.023 \pm 0.005$ & 0.023 \\
\hline $\begin{array}{c}\text { Effect } \\
(\mathrm{k}=4)\end{array}$ & - & - & $1.7 \cdot 10^{-5}$ \\
\hline
\end{tabular}

The data reported in Table 1 confirmed that methodological approach was accurate, but the effect was not detected and it is not possible under given conditions.

An analysis of Eq. (10) and Eq. (11) showed that for detecting cooperative annihilation - it is necessary to have a positron source with higher activity, as well as the system configuration with smaller solid angle from the geometric center to individual detectors.

In this experiment diameter of introduced lead collimator was $80 \mathrm{~mm}$. However, with a smaller diameter of the collimators (of $10 \mathrm{~mm}$, for example; reducing the detector's sensitive surface by a factor of $1.56 \cdot 10^{-2}$ ) and ${ }^{22} \mathrm{Na}$ with an activity of $4 \cdot 10^{8} \mathrm{~Bq}$, under the same conditions, background counting rate ( $\mathrm{N}_{\text {background }}$ ) would be $5.6 \mathrm{~s}^{-1}$, while the effect counting rate $\left(\mathrm{N}_{\text {effect }}\right)-0.26 \mathrm{~s}^{-1}$, i.e., the effect/background ratio would be around $4.6 \%$, enabling an isolation of cooperative annihilation from the background.

Moreover, by using the same positron source, and decreasing the detector's sensitive surface by a collimator of $3 \mathrm{~mm}$ in diameter, the counting rates would be $\mathrm{N}_{\text {background }}=4.5 \cdot 1 \mathrm{O}^{-2} \mathrm{~s}^{-1}$ and $\mathrm{N}_{\text {effect }}=2.4 \cdot 1 \mathrm{O}^{-2} \mathrm{~s}^{-}$ 1, i.e., the effect/background ratio of $\sim 53 \%$ - ensuring registration of the $p$-Ps cooperative annihilation (if actually exists).

Additionally, a use of positron beams (that increase counting rates of both - effect and background) will provide significantly shorter measuring time, since there is no registration of the $1275 \mathrm{keV}$ nuclear photon. 


\section{Conclusions}

Research on the cooperative decay is significant in the view of testing an interaction of Ps atoms in radiation fields.

Probability ratio - cooperative versus free annihilation of $p$-Ps, was evaluated to be $5 \cdot 10^{-5}$, with the effect/background ratio depending on the experimental setup photoefficiencies for the 511 and $1022 \mathrm{keV}$ photons (0.63 and 0.52 , respectively - at the ARGUS spectrometer). In the experiment carried out using ARGUS with collimators (80 $\mathrm{mm}$ in diameter), and ${ }^{22} \mathrm{Na}$ as a positron source (photoefficiency for the $1275 \mathrm{keV}$ - 0.49), background process (four-fold coincidences) obstructed detection of the effect. An analysis showed that the source activity was too low, but also diameter of the disk-shaped lead collimator too large.

Therefore, the cooperative $p$-Ps decay might be registered by the ARGUS spectrometer for a positron source $\left({ }^{22} \mathrm{Na}\right)$ with an activity of $4 \cdot 10^{8} \mathrm{~Bq}$, and diskshaped lead collimators mounted on each detector with a diameter of $\leq 10 \mathrm{~mm}$ (instead of $80 \mathrm{~mm}$ used in this study).

Finally, a use of positron beams, when registration of the $1275 \mathrm{keV}$ nuclear photon (from the ${ }^{22} \mathrm{Na}$ decay) is avoided, would provide shortening of measurement times.

Acknowledgements: The paper is a part of the research done within the projects supported by Ministry of Science of Montenegro, and Fundamental Research Foundation of the Belarusian Academy of Sciences.

\section{REFERENCES}

1. В.И. Гольданский, Физическая химия позитрона и позитрония, Москва, Россия: Нука, 1968. (V.I. Goldanskii, Physical Chemistry of Positron and Positronium, Moscow, Russia: Nauka, 1968.)

2. M. Chiba, R. Hamatsu, T. Hirose, T. Matsumoto and J. Yang, "Measurement of Electron-Positron Annihilation at Rest into Four and Five Photons," Nukleonika, vol. 42, no.1, pp. 61-68, Jan. 1997.

3. S.K. Andrukhovich et al., "Investigation of Orthopositronium $3 \gamma$-Decay Using a Multidetector Spectrometer," Nucl. Instrum. Meth. Phys. Res. Sec. B: Beam Int. Mat. Atoms, vol. 207, no. 2, pp. 219-226, June 2003.

4. B.K. Arbic, S. Hatamian, M. Skalsey, J. van House, W. Zheng, "Angular-Correlation Test of CPT in Polarized Positronium,” Phys. Rev., vol. 37, no. 9, pp. 3189-3194, 1988.

5. S.K. Andrukhovich, N. Antovich, A.V. Berestov and O.N. Metelitsa, "Test CPT in the Decay of Polarized Positronium Using Multidetector spectrometer," Mater. Sci. For., vol. 363-365, pp. 591-593, Apr. 2001.

6. P.A. Vetter and S.J. Freedman, "Search for CPT-Odd Decays of Positronium," Phys. Rev. Lett., vol. 91, no. 2631, p. 263401, Dec. 2003.

7. Р.А. Власов, О.Н. Гадомский, В.В. Самарцев,
"Аннигиляционное сверхизлучение в системе атомов позитрония и позитронная поляризация среды,” Теоретическая и математическая физ., т. 79, №. 3, c. 423-436, 1989. (R.A. Vlasov, O.N. Gadomskii and V.V.Samartsev, "Annihilation Superradiance in a System of Positronium Atoms and Positron Polarization of the Medium," Theor. Math. Phys., vol. 79, no. 3, pp. 423-436, 1989.)

8. O.N. Gadomskii, "The Positronium Atom in the Field of an Optical Laser: Radiation-Induced Energy Shifts and Annihilation Decay Kinetics," J. Exp. Theor. Phys., vol. 83, no. 4, pp. 676-684, Sep. 1996.

9. O.N. Gadomskii and T.T. Idiattulov, "Long-Lived Positro-nium Atom in the Field of an Optical Laser," Quant. Electron., vol. 28, no. 6, pp. 469-473, 1998.

10. L. B. Madsen and P. Lambropoulos, "Scaling of Hydroge-nic Atoms and Ions Interacting with Laser Fields: Posi-tronium in a Laser Field," Phys. Rev. A, vol. 59, no. 6, pp. 4574-4579, June 1999.

11. L.B. Madsen, "Positronium in Laser Fields," Nucl. Instr. Meth. B: Beam Inter. Mat. Atoms, vol. 221, no.1, pp. 174-181, July 2004.

12. N. Antovic, "Investigation of Rare Positronium Decays on Multidetector Gamma-Coincidence Spectrometers," PhD thesis, Faculty of Physics, University of Belgrade, Serbia, 2000. (in Serbian).

13. С.К. Андрухович, А.В. Берестов, Ф.Е. Зязюля, Б.А. Марцынкевич, Е.А. Рудак, А.М. Хильманович, “Автоматизированна регистрирующа гамма-установка совпадений (АРГУС), препринты в ИФ АН БССР, №. 408-409, 1986. ИФ АН БСС (S.K. Andrukhovich, A. V. Berestov, F.E. Zyazyulya, B.A. Marcinkevich, E.A. Rudak and A.M. Hil'manovich, [Automated Device for Registration of Gamma-Coincidences (ARGUS)]," Preprint in IF AN BSS, no. 408-409, 1986.)

14. N.M. Antovic, S.K. Andrukhovich and A.V. Berestov, "A Contribution of the Compton Scattered Radiation from Mn-54 to Double Gamma Coincidences Spectra at the 32-Detector System," in Proc. RAD 2014, Nis, Serbia, pp. 127-130.

15. S.K. Andrukhovich, N.M. Antovich and A.V. Berestov, "Effect of Cascade Gamma-Radiation Summation Processes on the Precision of Calculating the Probability of Three-Photon Annihilation of Positronium," Phys. Sol. State, vol. 42, но. 9, pp. 1648-1651, Sep. 2000. 